\title{
Intraoperative Injection of Radiocolloid for Sentinel Node Biopsy in Breast Cancer
}

\author{
Huan N. Vu ${ }^{1}$, Patricia F. O’Connor ${ }^{1}$, Rebecca R. Shoemaker ${ }^{2}$, Wen $W^{3}{ }^{3}$, Melvin J. Fratkin ${ }^{4}$, and Harry D. Bear ${ }^{5}$ \\ ${ }^{1}$ Virginia Commonwealth University, Richmond, Virginia; ${ }^{2}$ Wayne State University Detroit Medical Center, Detroit, Michigan; \\ ${ }^{3}$ Department of Biostatistics, Virginia Commonwealth University, Richmond, Virginia; ${ }^{4}$ Nuclear Medicine, Department of Radiology, \\ Virginia Commonwealth University, Richmond, Virginia; and ${ }^{5}$ Surgical Oncology, Department of Surgery, Virginia Commonwealth \\ University, Richmond, Virginia
}

Preoperative injection of radiocolloid for axillary sentinel node biopsy in breast cancer is uncomfortable for patients. This study evaluated the reliability of radiocolloid injection as determined by sentinel node identification rate and positive sentinel node biopsy rate, after the patient has been anesthetized in the operating room as compared with preoperative injection. Methods: After institutional review board approval, a retrospective cohort of patients with breast cancer who underwent sentinel node biopsy from January 2005 through December 2010 was evaluated for analysis. Patients who received intraoperative injection of radiocolloid were compared with those who received their injection preoperatively. Patients with incomplete pathologic staging or having received neoadjuvant chemotherapy were excluded. All patients received radiocolloid injections into the retroareolar tissue; some also received intradermal injection directly over the tumor. All injections contained $37 \mathrm{MBq}(1 \mathrm{mCi})$ in $0.5 \mathrm{~mL}$ of filtered $(0.22-\mu \mathrm{m})$ 99mTc-sulfur colloid. Results: Over the 6-y study period there were 904 sentinel node biopsy procedures, and 165 patients were excluded from analysis. Of the 739 sentinel node biopsies that were analyzed, 647 had preoperative injection of radiocolloid and 92 had intraoperative injection. The overall sentinel lymph node identification rates were similar for the 2 groups: $93.5 \%$ for the preoperative injection group and $94.6 \%$ for the intraoperative injection group (not statistically significant). The identification rates remained comparable for both groups when analyzed by $T$ stage of the tumor. The average number of sentinel lymph nodes removed was similar between preoperative and intraoperative injection: 2.60 and 2.70 nodes, respectively. The overall rates of positive sentinel nodes were comparable for the 2 groups: $25.6 \%$ for the preoperative injection group and $26.4 \%$ for the intraoperative injection group (not statistically significant). When analyzed by $\mathrm{T}$ stage, the positive sentinel node rates remained similar between the 2 groups. Conclusion: The sentinel lymph node identification rate of $94.6 \%$ for the intraoperative injection group was similar to other published sentinel lymph node identification rates (96\%-100\%). The positive sentinel lymph node rate was also comparable to that of published series. Intraoperative injection of radiocolloid for axillary sentinel node biopsy appears equivalent to preoperative injection and is a less painful experience for breast cancer patients.

\footnotetext{
Received Jul. 16, 2013; revision accepted Oct. 17, 2013.

For correspondence or reprints contact: Huan N. Vu, Virginia Commonwealth University, P.O. Box 980011, Richmond, VA 23298.

E-mail: hnvu@vcu.edu

Published online Nov. 14, 2013.

COPYRIGHT (C 2013 by the Society of Nuclear Medicine and Molecular Imaging, Inc.
}

Key Words: breast cancer; sentinel node biopsy; intraoperative injection; preoperative injection

J Nucl Med Technol 2013; 41:263-267

DOI: $10.2967 /$ jnmt.113.129460

$\mathbf{F}$ or breast cancer, the axillary nodal status has been crucial in determining the prognosis and indication for adjuvant systemic chemotherapy and adjuvant locoregional radiation therapy. Routine axillary nodal staging with axillary dissection, however, has not affected survival but has been associated with increased risk for ipsilateral upper-extremity lymphedema. To avoid nontherapeutic axillary dissection and reduce the incidence of lymphedema, axillary sentinel node biopsy has become the standard of care for axillary nodal staging of breast cancer. With sentinel node biopsy, only a few axillary nodes are selected and excised for pathologic evaluation rather than the anatomically directed axillary dissection. The preparation for sentinel node biopsy most frequently requires a preoperative injection of radiocolloid. A handheld radiation probe is then used intraoperatively to identify any radioactive sentinel nodes for excision.

To permit sufficient time for the injected radiocolloid to travel from the injection site to the axillary sentinel node, the radiocolloid is injected before the patient is anesthetized for the operation. Because the injection of radiocolloid is directed into the ipsilateral breast and frequently into the retroareolar space, the procedure is visibly uncomfortable for most patients. To reduce the pain experienced, some investigators have proposed performing the radiocolloid injection after the patient has undergone anesthesia. This study sought to determine whether injection of the radiocolloid once the patient has been anesthetized is comparable to preoperative injection for sentinel lymph node identification.

\section{MATERIALS AND METHODS}

This was a noninferiority retrospective study. The primary objective was to compare the sentinel node identification rate between patients with intraoperative injection of radiocolloid and those with preoperative injection. The hypothesis was that the identification rate of sentinel nodes in patients with intraoperative injection is 
not lower than the rate in those with preoperative injection. The secondary objective was to compare the positive sentinel node rate between preoperative and intraoperative injection. After we received institutional review board approval to collect data, we identified eligible patients through the Massey Cancer Center patient registry. The inclusion criterion was any patient who underwent sentinel node biopsy for breast cancer at Virginia Commonwealth University Health System (VCUHS) from January 1, 2005, through December 31, 2010. Patients whose records were incomplete with regard to breast cancer stage and sentinel biopsy were excluded. Patients who were treated with neoadjuvant chemotherapy before sentinel node biopsy were also excluded.

Injection with $37 \mathrm{MBq}(1 \mathrm{mCi})$ in $0.5 \mathrm{~mL}$ of filtered $(0.22-\mu \mathrm{m})$ ${ }^{99 \mathrm{~m}} \mathrm{Tc}$-sulfur colloid is used for sentinel node mapping at VCUHS. The site of radiocolloid injection for sentinel node localization varies among the 5 surgical oncologists who were active during the study period. All surgeons inject in the subareolar location; some surgeons also include peritumoral or intradermal injection of the radiocolloid. All but one surgeon usually injects the radiocolloid agent before anesthesia in the preoperative holding area. One surgeon routinely injects after induction of anesthesia in the operating room; other surgeons occasionally follow this procedure as well. Five milliliters of isosulfan blue (Lymphazurin; Covidien) are also routinely injected before surgical incision for visual localization of the sentinel nodes. Intraoperative audio localization of the sentinel node uses the C-Trak Automatic Analyzer system and the OmniProbe with collimator (Care Wise Medical Products Corp.).

Sentinel nodes are defined as hot and blue, hot but not blue, or not hot but blue. A hot sentinel node is defined a one having a count at least 10 times greater than the background count. The term hot is further defined as nodal tissue with more than $10 \%$ of the ex vivo count of the hottest sentinel node. The count value of each sentinel node itself is not routinely recorded otherwise. For this study, we based the sentinel lymph node identification rate on identification of at least one sentinel node in a patient who underwent sentinel node biopsy, and we obtained the pathologic T stage (pT) and pathologic N stage from the pathology record of the surgical specimen.

The statistical hypothesis was that the sentinel node identification rate with intraoperative injection (p1) would not be lower than the sentinel node identification rate with preoperative injection (p0). On the basis of clinical experience, the acceptable identification rate in patients with preoperative injection was set at $95 \%$ and a rate $85 \%$ or lower was considered unacceptably low. Therefore, the null hypothesis, $\mathrm{H} 0$, was $\mathrm{p} 0-\mathrm{p} 1 \geq 10 \%$, and the alternative hypothesis, $\mathrm{H} 1$, was $\mathrm{p} 0-\mathrm{p} 1<10 \%$. With use of a 2-proportion $z$ test, a minimum total of 118 patients with 59 in each group was deemed sufficient to gain $80 \%$ power at a type I error of 0.05 . The Fisher exact test was used to compare the 2 injection groups in terms of binary demographic characters, the identification rate overall and by $\mathrm{pT}$, and the rate of positive sentinel nodes overall, by $\mathrm{pT}$, and by axillary dissection. The 2-sample $t$ test was used to compare the number of sentinel nodes by pT and numeric demographic characters. A 2-sided type I error of 5\% was used for each test to define statistical significance. Statistical analysis used Prism 5.0 (GraphPad Software Inc.). In addition, a biostatistician reviewed all statistical analyses.

\section{RESULTS}

We identified for analysis 904 sentinel node biopsies that were performed for breast cancer at VCUHS between
January 1, 2005, and December 31, 2010. Of these, 35 were excluded because of incomplete information about the operation and cancer stage. A further 130 were excluded because the patients had received neoadjuvant chemotherapy before the sentinel node biopsy. The remaining 739 sentinel node biopsies were included in this analysis, with 647 having received preoperative injection of radiocolloid for localization and 92 having received intraoperative injection. The average age of the study population was $56.8 \mathrm{y}: 57.1 \mathrm{y}$ in the preoperative injection group and $53.4 \mathrm{y}$ in the intraoperative injection group. As expected, most patients were women (733 of the 739 biopsies). There were more Caucasian patients in the preoperative group $(60.0 \%$ vs. $34.8 \%)$ and fewer African-Americans (37.9\% vs. 62.0\%); the difference in ethnicity was statistically significant in both study groups $(P<0.0001)$. There were also more T1 tumors in the preoperative injection group $(65.5 \%$ vs. $51.1 \% ; P=0.0104)$ and fewer T2 tumors (22.1\% vs. $32.6 \%$; $P=0.0345)$. When the T1 tumors were subdivided into Tm, T1a, T1b, and T1c, only the T1c distribution was statistically different (36.5\% vs. $21.7 \% ; P=0.0049$ ); there were more T1c tumors in the preoperative injection group (Table 1).

The overall sentinel lymph node identification rates were comparable in the 2 groups (Fig. 1), 93.5\% for the preoperative injection group and $94.6 \%$ for the intraoperative injection group (not statistically significant). When analyzed by ethnicity, the identification rate of sentinel nodes in AfricanAmerican women (92.7\% and 91.2\%) and Caucasian women (94.1\% and $100 \%$ ) did not significantly differ between the preoperative and intraoperative injection groups. The identification rate remained equivalent for both groups when analyzed by the pT of the tumor: $92.6 \%$ vs. $91.7 \%$ for Tis, $93.4 \%$ vs. $97.9 \%$ for $\mathrm{T} 1$, and $94.4 \%$ vs. $90.0 \%$ for $\mathrm{T} 2$ in the preoperative group vs. the intraoperative group. Similarly, the average number of sentinel lymph nodes removed did

TABLE 1

Patient Demographics

\begin{tabular}{lccc}
\hline Demographic & $\begin{array}{c}\text { Preoperative } \\
\text { injection }\end{array}$ & $\begin{array}{c}\text { Intraoperative } \\
\text { injection }\end{array}$ & $P$ \\
\hline Total patients & 647 & 92 & \\
Age (y) & 57.1 & 53.4 & $\mathrm{NS}$ \\
Female:male & $641: 6$ & $92: 0$ & $\mathrm{NS}$ \\
Ethnicity & & & \\
Caucasian & $388(60.0)$ & $32(34.8)$ & $<0.0001$ \\
African-American & $245(37.9)$ & $57(62.0)$ & $<0.0001$ \\
Other & $14(2.2)$ & $3(3.3)$ & $\mathrm{NS}$ \\
pT stage & $68(10.5)$ & $12(13.0)$ & $\mathrm{NS}$ \\
Tis & $424(65.5)$ & $47(51.1)$ & 0.0104 \\
T1 & $143(22.1)$ & $30(32.6)$ & 0.0345 \\
T2 & $11(1.7)$ & $3(3.2)$ & $\mathrm{NS}$ \\
T3 & $1(0.2)$ & 0 & $\mathrm{NS}$ \\
T4 & & & \\
\end{tabular}

NS = not statistically significant.

Unless otherwise indicated, data are number of patients followed by percentage. 


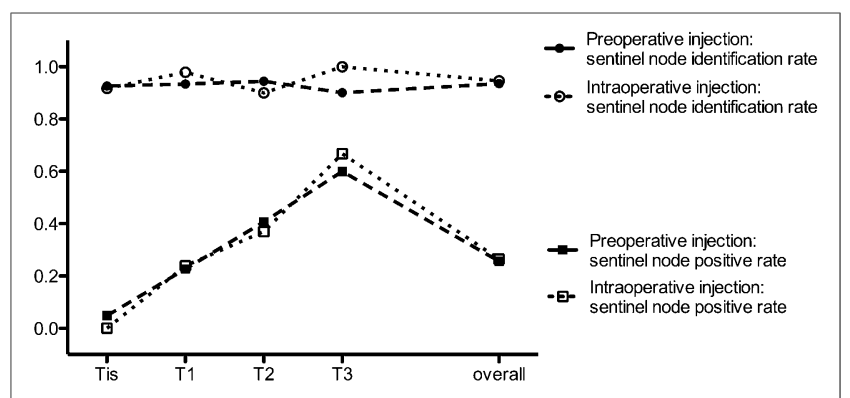

FIGURE 1. Sentinel node biopsy: preoperative vs. intraoperative injection.

not differ between the preoperative and the intraoperative groups: 2.60 nodes versus 2.70 nodes overall, 2.22 nodes versus 2.91 for Tis, 2.66 nodes versus 2.89 for T1, and 2.62 nodes versus 2.30 for T2 (Table 2).

The overall rates of positive sentinel nodes were comparable in the 2 groups (Fig. 1), 25.6\% for the preoperative injection group and $26.4 \%$ for the intraoperative injection group (not statistically significant). There was also no difference in the rate of biopsies positive for a sentinel node in African-American women (21.3\% and $28.1 \%$, respectively) or Caucasian women $(25.8 \%$ and $18.8 \%$, respectively) between the preoperative and intraoperative injection groups. When analyzed by $\mathrm{pT}$, the positivity rate remained similar: for T1 tumors, $22.7 \%$ in the preoperative group versus $23.9 \%$ in the intraoperative group; for T2 tumors, $40.7 \%$ versus $37.0 \%$, respectively (Table 3 ).

In the preoperative injection group, 42 patients had no sentinel node identified, 39 of whom went on to axillary dissection. Of these 39, 12 were found to have nodal metastasis, for a positive axilla rate of $30.8 \%$ on dissection. In the intraoperative injection group, all 5 patients in whom no sentinel node was found went on to axillary dissection. Of these 5, 3 had axillary nodal metastasis, for a positive axilla rate of $60.0 \%$ on dissection. These differences are not statistically significant.

\section{DISCUSSION}

The ability to perform selective axillary dissection for breast cancer through axillary sentinel lymph node biopsy has transformed the surgical care of patients with breast cancer. As a result, the incidence of nontherapeutic axillary dissection and its morbid consequences has been reduced. Radiocolloid detection is considered by most breast surgeons to be essential for sentinel lymph node biopsy. When a handheld radiation probe is used intraoperatively, audio signaling facilitates the detection of the radioactive sentinel node in the axilla. The agent used, ${ }^{99 \mathrm{~m} T c-s u l f u r}$ colloid, has an average diameter of about $0.2 \mu \mathrm{m}$; larger particles may be too large for lymphatic mapping. Only the axilla is explored for sentinel mapping in breast cancer, as axillary nodal staging remains an essential component in guiding breast cancer care. Although breast cancer can metastasize to the internal mammary nodes, the overall incidence is low and the instances of internal mammary node metastasis without axillary metastasis are even lower (1). Routine sampling of the internal mammary nodes has largely been abandoned.

The use of radiocolloid for operative identification of the sentinel node has, however, introduced a new morbidity: the pain associated with the preoperative retroareolar injection. Chandler et al. reported that in $35 \%$ of patients, the injection of radiocolloid was associated with significant pain, which occurred regardless of the use or timing of anesthetic cream (2). In a study of 39 patients randomized to treatment groups receiving topical lidocaine/prilocaine cream versus placebo before radiocolloid injection, the addition of local anesthesia failed to reduce the perceived pain associated with the injection (3). In a prospective randomized study of 140 patients, the addition of $1 \%$ lidocaine to the radiocolloid solution decreased the perceived pain but was associated

TABLE 2

Sentinel Node Identification

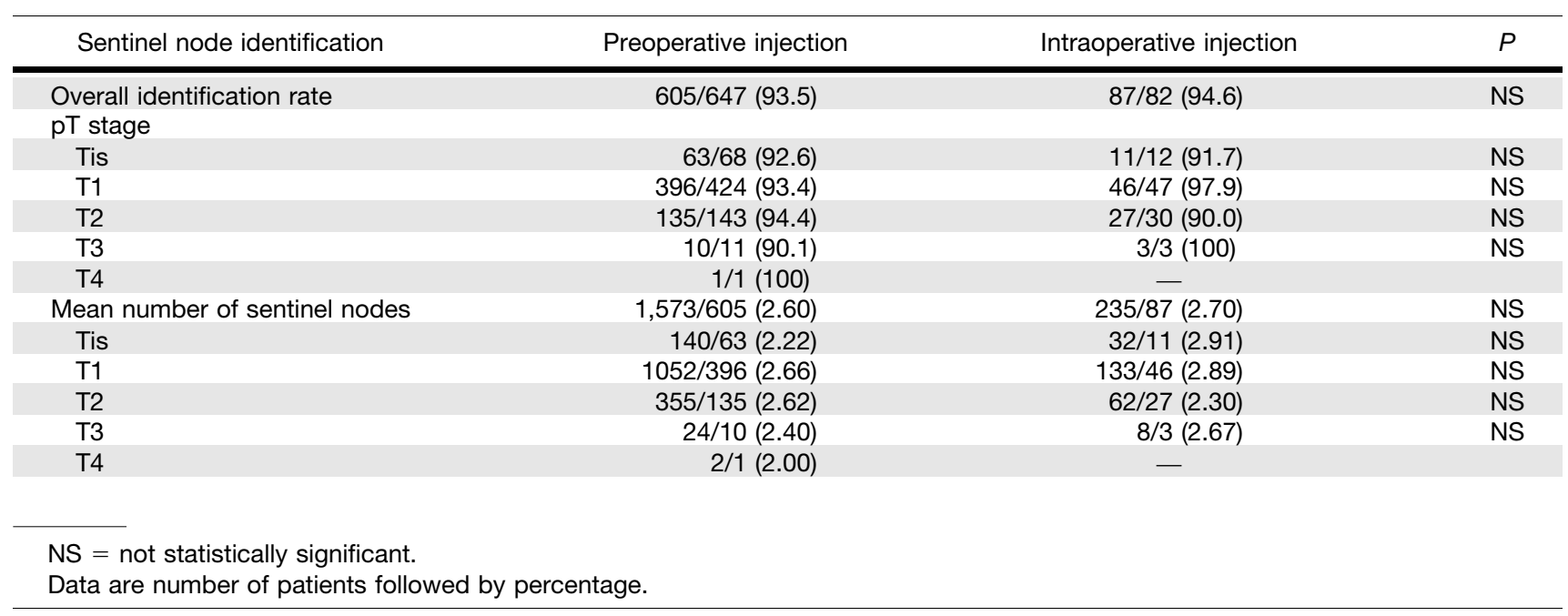


TABLE 3

Sentinel Node Malignancy Rate

\begin{tabular}{cccc}
\hline Parameter & $\begin{array}{c}\text { Preoperative } \\
\text { injection }\end{array}$ & $\begin{array}{c}\text { Intraoperative } \\
\text { injection }\end{array}$ & $P$ \\
\hline $\begin{array}{c}\text { Positive sentinel } \\
\text { biopsy }\end{array}$ & $155 / 605(25.6)$ & $23 / 87(26.4)$ & NS \\
pT stage & & & \\
Tis & $3 / 63(4.8)$ & $0 / 11(0)$ & NS \\
T1 & $90 / 396(22.7)$ & $11 / 46(23.9)$ & NS \\
T2 & $55 / 135(40.7)$ & $10 / 27(37.0)$ & NS \\
T3 & $6 / 10(60.0)$ & $2 / 3(66.7)$ & NS \\
T4 & $1 / 1(100)$ & - & \\
\hline
\end{tabular}

NS $=$ not statistically significant.

Data are number of patients followed by percentage.

with detection of fewer sentinel lymph nodes, even though the overall rate of sentinel node identification did not change (4). Radowsky also noted that providers tended to underestimate the level of pain the patients experienced when the patient reported the highest pain rating (5). Clearly, if the injection of radiocolloid can occur after the patient has been anesthetized in the operating room, the pain of radiocolloid injection will be eliminated.

The concern about intraoperative injection of radiocolloid is whether there would be sufficient time for the radiocolloid to travel from the injection site to the sentinel lymph nodes. Injection of radiocolloid the day of the biopsy appears to be equivalent to injection the day before, in terms of the false-negative sentinel node rate and the number of sentinel nodes identified (6). A study by Wondergem et al. of 117 patients who underwent radiocolloid injection and lymphoscintigraphy 1 and $2 \mathrm{~h}$ later suggests decreased sentinel lymph node localization at $1 \mathrm{~h}$ when compared with $2 \mathrm{~h}$. At $1 \mathrm{~h}$, lymphoscintigraphy localized a sentinel node in $79.5 \%$ of patients, with an increase to $95.7 \%$ at $2 \mathrm{~h}$ (7). However, localization by lymphoscintigraphy may not be as sensitive as that by operative exploration using a handheld probe and blue dye. Although lymphoscintigraphy can detect sentinel nodes outside the axilla, nonaxillary nodal activities would not be surgically relevant because only the axilla is explored at surgery.

Other surgical groups have reported their experience with intraoperative injection of radiocolloid for sentinel node biopsy. Layeeque et al., in performing 96 sentinel node biopsies using $39.6 \mathrm{MBq}(1.07 \mathrm{mCi})$ of unfiltered ${ }^{99 \mathrm{~m}} \mathrm{Tc}$-sulfur colloid and blue dye injected intraoperatively into the retroareolar location, found a sentinel node localization rate of $97 \%$ (8). Zogakis et al., in performing 122 sentinel node biopsies using $37-74 \mathrm{MBq}(1-2 \mathrm{mCi})$ of filtered ${ }^{99 \mathrm{~m}} \mathrm{Tc}$-sulfur colloid and isosulfan blue (also injected intraoperatively into the retroareolar location), had a $99.2 \%$ identification rate (9). Thompson et al. reported an identification rate of $96 \%$ for 252 patients with intraoperative injection of $4 \mathrm{~mL}$ of unfiltered ${ }^{99 \mathrm{~m}} \mathrm{Tc}$-sulfur colloid (37 MBq [1 mCi]) and isosulfan blue (10). Dauphine et al. prospectively randomized
100 patients into a preoperative injection group and 100 into an intraoperative injection group who received $5 \mathrm{~mL}$ of filtered ${ }^{99 \mathrm{~m} T c-s u l f u r}$ colloid (18.5 MBq [0.5 mCi] $)$ and blue dye. The 2 groups had equivalent sentinel lymph node identification rates: $96 \%$ for preoperative injection and $100 \%$ for intraoperative injection (11). Dixon et al. reported a similar identification rate of $98 \%$ for 163 sentinel node biopsies using $0.5 \mathrm{~mL}$ of ${ }^{99 \mathrm{~m}} \mathrm{Tc}$ sulfur colloid $(25 \mathrm{MBq}$ [0.68 mCi]) and blue dye intraoperatively injected into the retroareolar location (12). Johnson et al. expanded an experiment of Layeeque et al. (8) to include 775 sentinel node biopsies with $37 \mathrm{MBq}(1 \mathrm{mCi})$ of ${ }^{99 \mathrm{~m}} \mathrm{Tc}$ sulfur colloid, mostly without blue dye. The sentinel node identification rate remained excellent at $98.6 \%$ (13). The VCUHS study has found equivalent sentinel lymph node identification rates for both groups, $93.5 \%$ for preoperative injection and $94.6 \%$ for intraoperative injection. The rate of sentinel lymph node identification with intraoperative injection at VCUHS appears similar to that of the previously mentioned publications $(96 \%-100 \%)$.

Despite the acceptable sentinel node identification rate, there naturally remain concerns that the identified node may not represent the oncologic sentinel lymph node. Without all patients undergoing axillary dissection, the true falsenegative sentinel node biopsy rate cannot be determined. However, an analysis of the positive sentinel node biopsy rate with published pTs suggests comparability between the 2 techniques. Stell et al. compared the incidence of positive sentinel lymph nodes between 102 patients with preoperative injection of radiocolloid and that of 112 patients with intraoperative injection. The 2 groups had equivalent tumor characteristics and incidence of positive sentinel lymph nodes, $20.6 \%$ for preoperative injection versus $19.6 \%$ for intraoperative injection (14). The VCUHS study also shows no statistical difference in the overall positive sentinel node biopsy rate between preoperative injection $(25.6 \%)$ and intraoperative injection (26.4\%). This study, however, has fewer $\mathrm{T} 1$ and more $\mathrm{T} 2$ tumors in the intraoperative injection group $(51.1 \%$ are $\mathrm{T} 1$ and $32.6 \%$ are $\mathrm{T} 2)$ than in the preoperative injection group (65.5\% are $\mathrm{T} 1$ and $22.1 \%$ are $\mathrm{T} 2)$. The study of Stell et al. did not break the patient population into ethnic groups but did have an equivalent distribution of tumor stage. When analyzed by pTs, the sentinel node positive rate did not differ between the intraoperative and preoperative injection groups for T1 (23.9\% vs. $22.7 \%)$ or for $\mathrm{T} 2(37.0 \%$ vs. $40.7 \%)$. In addition, the $23.9 \%$ positive sentinel node biopsy rate for $\mathrm{T} 1$ and $37.0 \%$ for $\mathrm{T} 2$ with intraoperative injection in the VCUHS series are comparable to those of other intraoperative injection series. Zogakis et al. reported $16.8 \%$ positive sentinel node biopsies for $\mathrm{T} 1$ and $44 \%$ for T2 (9). The series of Thompson et al. had an $11 \%$ positive sentinel node biopsy rate for $\mathrm{T} 1$ and $25 \%$ for $\mathrm{T} 2$ (10). The positivity rate of the VCUHS experience with intraoperative injection of $\mathrm{T} 2$ tumors falls neatly between those of Zogakis and Thompson, and the positivity rate of T1 tumors exceeds both, thus decreasing the chances of a false-negative sentinel node biopsy. 
Because the study was not prospective and randomized, there are several limitations. First, the 2 study groups may not be comparable because they differed demographically. How the difference in ethnicity might affect the outcome remains unclear, but once the findings were stratified by ethnicity, there did not appear to be any difference in sentinel node identification or positive sentinel nodes. Second, there was no standardization of injection site for sentinel node biopsy; according to the surgeon's preference, some patients underwent peritumoral or intradermal injection as well as retroareolar injection. Randomized studies, however, have endorsed the retroareolar injection site, used in all studied patients, as reliable (15-18). Because the injection site has not appeared to affect sentinel node identification in the literature, differences in injection site may not be significant. Third, the time from radiocolloid injection to surgical incision was not quantified for any of the studied patients. Empirically, there is about an hour from injection to incision for the preoperative group and $15 \mathrm{~min}$ for the intraoperative group. Finally, there was no true-negative control for analysis, leaving the possibility that despite equivalence of observed sentinel node identification rate, the true sentinel node identification rate might be different. Tumor stagematched analysis of positive sentinel node biopsy, which appears comparable between the 2 groups, was used instead. Despite the limitations of this study, the findings do support those of other published studies endorsing equivalence between intraoperative and preoperative injection of radiocolloid for sentinel node biopsy in breast cancer.

\section{CONCLUSION}

Intraoperative injection of radiocolloid appears to produce an acceptable sentinel node biopsy identification rate, compared with that of preoperative injection. The main benefit is the reduction of discomfort, as the injection of radiocolloid occurs under operative anesthesia. An additional benefit is a 1-step process, as the patient would present for a combined sentinel node injection, sentinel node mapping, sentinel node biopsy, and breast surgical therapy. Currently, many surgical practices for breast cancer arrange for sentinel node radiocolloid injection the day before the operation to facilitate morning sentinel node biopsy and to minimize any delays in the start of surgery. Intraoperative injection would still require that the radiocolloid be available for injection the morning of the operation. The implementation of intraoperative injection requires a partnership among the department of surgery, the department of nuclear medicine, and the operating room services to maintain radiation safety standards.

\section{DISCLOSURE}

No potential conflict of interest relevant to this article was reported.

\section{ACKNOWLEDGMENT}

We thank Jenni Brumelle, $\mathrm{PhD}$, for her scientific review of the manuscript.

\section{REFERENCES}

1. Mansel RE, Goyal A, Newcombe RG; ALMANAC Trialists Group. Internal mammary node drainage and its role in sentinel lymph node biopsy: the initial ALMANAC experience. Clin Breast Cancer. 2004;5:279-284.

2. Chandler KJ, Hunt $\mathrm{CH}$, Morreale R, et al. Effect of patient age, breast density, and topical anesthetic cream on perceived pain with sentinel lymph node scintigraphy. J Nucl Med Technol. 2012;40:44-47.

3. O'Connor JM, Helmer SD, Osland JS, et al. Do topical anesthetics reduce periareolar injectional pain before sentinel lymph node biopsy? Am J Surg. 2011; 202:707-711.

4. Arciero CA, Henry LR, Howard RS, et al. Technical effects of adding $1 \%$ lidocaine to technetium sulfur colloid for sentinel lymphatic mapping in early breast cancer: analysis of data from a double-blind randomized controlled trial. Ann Surg Oncol. 2013;20:2548-2555.

5. Radowsky JS, Baines L, Howard RS, et al. Pain ratings by patients and their providers of radionucleotide injection for breast cancer lymphatic mapping. Pain Med. 2012;13:670-676.

6. Ali J, Alireza R, Mostafa M, et al. Comparison between one day and two days protocols for sentinel node mapping of breast cancer patients. Hell J Nucl Med. 2011;14:313-315.

7. Wondergem M, Hobbelink MG, Witkamp AJ, et al. Breast cancer sentinel node scintigraphy: differences between imaging results 1 and $2 \mathrm{~h}$ after injection. Nucl Med Commun. 2012;33:1138-1143.

8. Layeeque R, Kepple J, Henry-Tillman RS, et al. Intraoperative subareolar radioisotope injection for immediate sentinel lymph node biopsy. Ann Surg. 2004;239:841-845.

9. Zogakis TG, Wetherille RE, Christensen RD, et al. Intraoperative subareolar injection of ${ }^{99 \mathrm{~m} T c-l a b e l e d ~ s u l f u r ~ c o l l o i d ~ r e s u l t s ~ i n ~ c o n s i s t e n t ~ s e n t i n e l ~ l y m p h ~ n o d e ~}$ identification. Ann Surg Oncol. 2005;12:167-172.

10. Thompson M, Korourian S, Henry-Tillman R, et al. Intraoperative radioisotope injection for sentinel lymph node biopsy. Ann Surg Oncol. 2008;15:3216-3221

11. Dauphine CE, Khalkhali I, Vargas MP, et al. Intraoperative injection of technetium-99m sulfur colloid is effective in the detection of sentinel lymph nodes in breast cancer. Am J Surg. 2006;192:423-426.

12. Dixon JM, Mak C, Radhakrishna S, et al. Effectiveness of immediate preoperative injection of radiopharmaceutical and blue dye for sentinel node biopsy in patients with breast cancer. Eur J Cancer. 2009;45:795-799.

13. Johnson CB, Boneti C, Korourian S, et al. Intraoperative injection of subareolar or dermal radioisotope results in predictable identification of sentinel lymph nodes in breast cancer. Ann Surg. 2011;254:612-618.

14. Stell VH, Flippo-Morton TS, Norton HJ, White RL Jr. Effect of intraoperative radiocolloid injection on sentinel lymph node biopsy in patients with breast cancer. Ann Surg Oncol. 2009;16:2300-2304.

15. Smith LF, Cross MJ, Klimberg VS. Subareolar injection is a better technique for sentinel lymph node biopsy. Am J Surg. 2000;180:434-437.

16. Mateos JJ, Vidal-Sicart S, Zanón G, et al. Sentinel lymph node biopsy in breast cancer patients: subdermal versus peritumoural radiocolloid injection. $\mathrm{Nucl} \mathrm{Med}$ Commun. 2001;22:17-24.

17. Povoski SP, Olsen JO, Young DC, et al. Prospective randomized clinical trial comparing intradermal, intraparenchymal, and subareolar injection routes for sentinel lymph node mapping and biopsy in breast cancer. Ann Surg Oncol. 2006;13:1412-1421.

18. Rodier JF, Velten M, Wilt M, et al. Prospective multicentric randomized study comparing periareolar and peritumoral injection of radiotracer and blue dye for the detection of sentinel lymph node in breast sparing procedures: FRANSENODE trial. J Clin Oncol. 2007;25:3664-3669. 